\title{
Metabolismo en asociación a mortalidad: significado pronóstico de hipoalbuminemia y diabetes mellitus tipo 2 en el paciente hospitalizado con neumonía nosocomial
}

\author{
Liliana Alcaraz-Gaytán*, Rafael Luna-Montalbán, Érica García-Valadez y Ruth Gutiérrez-Padilla \\ Unidad Médica de Alta Especialidad 1, Centro Médico Nacional del Bajío, Instituto Mexicano del Seguro Social, León, Gto., México
}

\begin{abstract}
Background: Hypoalbuminemia is associated with the nutritional status of patients with type 2 diabetes mellitus (DM2); it is related to oxidative stress and alterations in lipid and protein metabolism, and is a risk factor for mortality in the critically ill patient. Furthermore, DM2 has been associated with an increase in infection-related mortality. We explored the association of these metabolic conditions with mortality and related factors in patients with nosocomial pneumonia. Methods: We included 120 patients with nosocomial pneumonia in a referral hospital; demographic and clinical data were obtained. Data were compared by $\chi^{2}$ and logistic regression analysis. Results and conclusions: Mortality of patients with hypoalbuminemia was 50\%; 43/46 (91.2\%) patients who died had hypoalbuminemia ( $p=0.0001$ ). DM2 was present in $59.2 \%$ of patients, and was significantly associated with hypoalbuminemia $(p<0.05)$. Other associated factors were mechanical ventilation $(91.7 \%)$, higher Candida Score, higher incidence of acute renal failure, and higher requirements of $\mathrm{FiO}_{2}$. Results showed that low albumin levels were an independent mortality factor. (Hosp Med Clin Manag. 2019;12:107-12)

Corresponding author: Liliana Alcaraz-Gaytán, Nexusyosoy@gmail.com
\end{abstract}

Key words: Diabetes mellitus 2. Nosocomial pneumonia. Mortality association. Hypoalbuminemia.

\section{RESUMEN}

Antecedentes: La hipoalbuminemia se asocia al estado nutricional de los pacientes con diabetes mellitus tipo 2 (DM2); correlaciona con estrés oxidativo y alteraciones en el metabolismo lipídico y proteico. Se ha estudiado la hipoalbuminemia como factor de mortalidad en el paciente grave, y también se ha asociado la DM2 con un incremento en la mortalidad en infecciones. El objetivo del estudio fue definir la asociación de estas condiciones metabólicas con mortalidad y factores asociados en pacientes con neumonía nosocomial. Métodos: Se incluyeron 120 pacientes hospitalizados con neumonía nosocomial en un hospital de referencia; se evaluaron variables sociodemográficas y clínicas y se hicieron análisis comparativo y de regresión logística. Resultados y conclusiones: La mortalidad fue del $50 \%$ en los pacientes con hipoalbuminemia, y del total de pacientes fallecidos el 91.2\% (43/46) presentaba

Correspondencia:

*Liliana Alcaraz-Gaytán

E-mail: Nexusyosoy@gmail.com
Date of reception: 24-04-2018

Date of acceptance: 19-08-2019

DOI: 10.24875/HMCM.19000120 
hipoalbuminemia $(p=0.0001)$. La DM2 se asoció significativamente $(p<0.05)$ con hipoalbuminemia, presentándose en el 59.2\%. Otros factores asociados fueron la ventilación mecánica (91.7\%), mayor Candida Score, insuficiencia renal aguda y mayores requerimientos de $\mathrm{FiO}_{2}$. Encontramos que los niveles bajos de albúmina fueron un factor independiente de mortalidad.

Palabras clave: Diabetes mellitus 2. Neumonía nosocomial. Asociación. Hipoalbuminemia. Mortalidad.

\section{INTRODUCCIÓN}

El estado metabólico de un paciente es difícil de estudiar, sobre todo cuando este se encuentra en estado crítico. Aunque se conoce la relación entre el estado nutricional y la mortalidad, es difícil darle el valor que le corresponde. Es ampliamente aceptado que los diabéticos tienen mayor propensión a desarrollar infecciones. La quimiotaxis de neutrófilos, adherencia al endotelio vascular, fagocitosis, actividad bactericida intracelular, opsonización y la inmunidad mediada por células están deprimidas en los diabéticos con hiperglucemia ${ }^{1,2}$. La hipoalbuminemia tiene un fuerte valor predictivo de mortalidad y morbilidad ${ }^{3}$. Estudios recientes establecen que concentraciones más bajas de albúmina se asocian con un mayor riesgo de enfermedad coronaria, mortalidad cardiovascular y aterosclerosis carotídea ${ }^{4,5}$.

Especies reactivas de oxígeno (ERO) es un término genérico en el que se incluyen radicales libres y compuestos no radicales libres derivados del oxígeno molecular, como el radical anión superóxido, radical hidroxilo y el peróxido de hidrógeno. El desequilibrio entre la producción de ERO y las defensas antioxidantes puede conducir a estrés oxidativo. El estrés oxidativo lleva a peroxidación lipídica, alteración de las propiedades de las membranas y de las proteínas allí insertadas, así como daño proteínico directo y del ADN mitocondrial ${ }^{6}$.

La albúmina tiene una vida media de 15 a 19 días y su catabolismo se lleva a cabo en el endotelio vascular?. Las concentraciones pueden descender rápidamente ante estrés, traumatismo o sepsis; esto se debe a la acelerada redistribución desde el espacio intravascular, disminución en su síntesis o aumento del catabolismo. Por lo anterior, la albúmina se ha relacionado directamente con el grado de inflamación ${ }^{8,9}$.

En este estudio se buscó la relación de la diabetes mellitus tipo 2 (DM2) con alteraciones metabólicas mediante su asociación con hipoalbuminemia en el paciente hospitalizado con neumonía nosocomial.

\section{MÉTODOS}

\section{Diseño y población de estudio}

Estudio longitudinal, prospectivo, abierto, efectuado de agosto del 2016 a diciembre del 2017 en 120 pacientes con neumonía nosocomial, a partir del programa de optimización en el uso de antimicrobianos en la Unidad Médica de Alta Especialidad (UMAE) 1 del Centro Médico Nacional del Bajío del Instituto Mexicano del Seguro Social, en León, Gto.

\section{Tamaño de la muestra}

Con base en el estudio de Gutiérrez, et al. ${ }^{10}$, quienes identificaron DM2 en el $18.14 \%$ de los pacientes hospitalizados, y con potencia del $80 \%$, precisión del $7 \%$ y significación del 95\%, se calculó el tamaño de muestra mediante el método de una proporción y odds ratios (OR) con riesgo alfa de 0.05 y beta 0.2 , y se determinó que se requerían 120 pacientes con neumonía nosocomial. El muestreo fue no probabilístico, por disponibilidad, hasta completar el tamaño de muestra; se incluyeron 46 mujeres y 74 hombres.

\section{Criterios de inclusión y exclusión}

Los participantes de este estudio fueron pacientes hospitalizados que cumplieron los siguientes criterios de inclusión: a) pacientes hospitalizados en la UMAE1, mayores de 16 años, sexo indistinto, y b) con criterios diagnósticos relativos a neumonía nosocomial definidos por la IDSA (Infectious Diseases Society of America). Los criterios de exclusión fueron: a) bronquitis aguda o diagnóstico de neumonía incierto; b) pacientes neutropénicos, inmunocomprometidos, embarazadas, con antibioticoterapia en los últimos 30 días. Y los criterios 
de eliminación: pacientes con complicaciones infecciosas, insuficiencia cardiaca o enfermedad que condicione a insuficiencia cardiaca aguda.

\section{Procedimientos}

Se registraron variables sociodemográficas y de condiciones comórbidas, como sexo, edad, estado civil, peso, índice de masa corporal (IMC), puntuación en la APACHE II (Acute Physiology And Chronic Health Evaluation II), días de ventilación mecánica, DM2, hipertensión arterial sistémica, insuficiencia renal aguda (IRA), hepatopatía crónica y uso de aminas. Se realizó bioquímica completa para determinar el estado metabólico de los pacientes, así como seguimiento del desenlace de cada paciente hasta la resolución del cuadro o muerte, considerando a lo largo de este escalas pronósticas, ventilación mecánica y complicaciones asociadas. Se consideró hipoalbuminemia a la disminución en los niveles séricos de albúmina por debajo de $3.5 \mathrm{~g} / \mathrm{dl}$.

\section{Aspectos éticos}

El proyecto fue autorizado por el comité local de investigación (R-2016-501-55). Se solicitó el consentimiento informado a los pacientes para su seguimiento. En todo caso se dio cumplimiento de los principios éticos expresados en la Declaración de Helsinki de la Asociación Médica Mundial y el Reglamento de la Ley General de Salud en México. Esta investigación fue aprobada por el Comité Local de Investigación y Ética en Investigación en Salud N. ${ }^{\circ}$ 501, con número de registro $13 \mathrm{Cl} 05$ 035202 ante COFEPRIS.

\section{Análisis estadístico}

Se realizó un muestreo no probabilístico, por disponibilidad. Se llevó registro de cada caso y se realizó un concentrado en hoja de cálculo; se calculó la proporción de pacientes con DM2, y su asociación con albumina sérica e hipoalbuminemia. Se caracterizó la población al final del estudio mediante estadística descriptiva. Se utilizó el paquete estadístico IBM SPSS ${ }^{\circledR}$ 2.1. Las variables cualitativas se compararon mediante $\chi^{2}$ o pruebas exactas, y las numéricas mediante la prueba $t$ de muestras independientes o $U$ de MannWhitney. Para comparar la proporción de errores entre ambos grupos se utilizó $\chi^{2}$ o prueba exacta. Se calcularon intervalos de confianza del $95 \%$ y un valor de alfa menor a 0.05 se consideró significativo.
Tabla 1. Variables sociodemográficas y clínicas de los pacientes con neumonía nosocomial

\begin{tabular}{lc}
\hline Variable & \\
\hline Edad, años (rango) & $56.31(18-93)$ \\
Sexo femenino & $46(38.3 \%)$ \\
Sexo masculino & $74(61.7 \%)$ \\
Peso, kg & $73.554 \pm 14.89$ \\
IMC & $27.35 \pm 5.23$ \\
Talla, m & $1.64 \pm 0.07$ \\
Creatinina & $1.64 \pm 2.46$ \\
BUN & $28.95 \pm 24.88$ \\
Albúmina, g/dl & $2.96 \pm 0.8$ \\
Depuración de creatinina en 24 h & $86.99 \pm 73$ \\
Superficie corporal & $1.81 \pm 0.207$ \\
APACHE II & $11.85 \pm 7.13$ \\
Mortalidad predicha & $11.78 \pm 7.1$ \\
Días de ventilación mecánica & $6 \pm 8.13$ \\
Leucocitos & $9.88 \pm 5.5$ \\
Saturación & $93.38 \pm 3.88$ \\
FiO ${ }_{2}$ & $43.18 \pm 19$ \\
Diabetes mellitus 2 & $49(40.8 \%)$ \\
Hipertensión arterial sistémica & $44(36.7 \%)$ \\
Insuficiencia renal aguda & $16(13.3 \%)$ \\
Hepatopatía crónica & $2(1.7 \%)$ \\
Aminas & $23(19.2 \%)$ \\
Hipoalbuminemia & $86(71.7 \%)$ \\
SIRS & $24(20 \%)$ \\
Fiebre & $46(38.3 \%)$ \\
\hline . & \\
\hline
\end{tabular}

IMC: índice de masa corporal; BUN: nitrógeno ureico en sangre; APACHE II: Acute Physiology And Chronic Health Evaluation II; $\mathrm{FiO}_{2}$ : fracción inspirada de oxigeno; SIRS: respuesta inflamatoria sistémica.

\section{RESULTADOS}

Se incluyeron 46 mujeres (38.3\%) y 74 hombres (61.7\%); de ellos, 49 (40.8\%) presentaban DM2. El rango del IMC fue de 16 a 41.4 , con una media de 27.35. La puntuación de APACHE II fue de 2 a 38, con un promedio de 11.85. La creatinina tuvo un rango de 0.1 a 18 , con una media de 1.64. El rango de días de ventilación mecánica fue de 0 a 36, con una media de 6 (Tabla 1).

Se encontraron distintos factores que afectaron significativamente la mortalidad, principalmente el hecho de tener ventilación mecánica asistida y con ello los días de ventilación mecánica, con mayor proporción de pacientes con desenlace fatal en aquellos con 9.6 días vs. 3.7 
Tabla 2. Factores relacionados a supervivencia y mortalidad

\begin{tabular}{|c|c|c|c|}
\hline Factor & $\begin{array}{c}\text { Sobrevivientes } \\
\text { n (\%) }\end{array}$ & $\begin{array}{c}\text { Fallecidos } \\
\text { n (\%) }\end{array}$ & $p$ \\
\hline Hipoalbuminemia & $43(50)$ & $43(50)$ & 0.0001 \\
\hline Albúmina normal & $31(91.2)$ & $3(8.8)$ & 0.0001 \\
\hline Ventilación mecánica & $24(32.4)$ & $36(78.3)$ & 0.0001 \\
\hline Sexo femenino & $27(36.5)$ & $19(41.3)$ & 0.598 \\
\hline Sexo masculino & $47(63.5)$ & $27(58.7)$ & 0.598 \\
\hline Child-Pugh B & $1(1.4)$ & $1(2.2)$ & 0.732 \\
\hline Diabetes mellitus & $35(47.3)$ & $14(30.4)$ & 0.068 \\
\hline Hipertensión arterial & $25(33.8)$ & 19 (41.3) & 0.406 \\
\hline Insuficiencia renal crónica & $8(10.8)$ & $8(17.4)$ & 0.303 \\
\hline Hepatopatía & $1(1.4)$ & $1(2.2)$ & 0.732 \\
\hline SIRS & $13(17.6)$ & $11(23.9)$ & 0.398 \\
\hline Fiebre & $25(33.8)$ & $21(45.7)$ & 0.194 \\
\hline IMC, media & 27.62 & 26.91 & 0.469 \\
\hline Edad, años & 54.53 & 59.17 & 0.176 \\
\hline Superficie corporal & 1.82 & 1.81 & 0.766 \\
\hline Días de VM, media & 3.77 & 9.60 & 0.0001 \\
\hline Creatinina, media & 1.49 & 1.89 & 0.236 \\
\hline BUN, media & 28.6 & 29.52 & 0.905 \\
\hline Albúmina, media & 3.21 & 2.55 & 0.0001 \\
\hline Depuración de creatinina 24 h & 89.16 & 83.50 & 0.066 \\
\hline APACHE II & 9.81 & 15.13 & 0.0001 \\
\hline CPIS & 4.21 & 6.61 & 0.0001 \\
\hline Riesgo general & 0.56 & 0.30 & 0.045 \\
\hline Riesgo de Staphylococcus & 0.41 & 0.50 & 0.997 \\
\hline Riesgo de Pseudomonas & 0.39 & 0.60 & 0.051 \\
\hline Cándida Score & 0.35 & 0.70 & 0.001 \\
\hline Leucocitos & 9.51 & 10.47 & 0.091 \\
\hline Saturación & 93.46 & 93.24 & 0.892 \\
\hline $\mathrm{FiO}_{2}$ & 36.34 & 54.2 & 0.0001 \\
\hline
\end{tabular}

Los resultados se expresan en media de los valores.

IMC: índice de masa corporal; BUN: nitrógeno ureico en sangre; APACHE II: Acute Physiology And Chronic Health Evaluation II; CPIS: escala de valoración clínica de la infección pulmonar; $\mathrm{FiO}_{2}$ : fracción inspirada de oxigeno ; SIRS: respuesta inflamatoria sistémica; VM: ventilación mecánica.

$(p=0.0001)$. También hubo diferencia en los niveles de albúmina, siendo menores en los pacientes que fallecieron, quienes tuvieron niveles de 2.55 vs. $3.21(p=0.0001)$. La puntuación APACHE II también fue mayor en el grupo con mayor mortalidad: 15.13 vs. 9.81. La puntuación CPIS (escala de valoración clínica de la infección pulmonar) de los pacientes fue mayor en los que presentaron mayor mortalidad: 6.6 vs. $4.2(p=0.0001)$. El Candida Score también tuvo mayores puntuaciones en este grupo, que también tuvo requerimientos mayores de fracción inspirada de oxigeno $\left(\mathrm{FiO}_{2}\right)(\mathrm{p}=0.0001)$ (Tabla 2).
Los pacientes con niveles normales de albúmina tuvieron una supervivencia del 91.2\% (31/34). En contraste, en los pacientes con hipoalbuminemia se presentó el mismo número de muertes que de sobrevivientes (46/46), y del total de muertes reportado, el $91.2 \%$ (43/46) presentaba hipoalbuminemia $(p=0.0001)$.

Los factores que se asociaron significativamente $(p<0.05)$ con hipoalbuminemia fueron: ventilación mecánica en el 91.7\%, DM2 en el 59.2\%, mayor Candida Score, mayor incidencia de IRA y mayores 
Tabla 3. Variables asociadas a hipoalbuminemia

\begin{tabular}{|c|c|c|c|}
\hline Variable & Albúmina normal & Hipoalbuminemia & p \\
\hline Mortalidad & $3(8.8 \%)$ & $43(91.2 \%)$ & 0.0001 \\
\hline Sexo femenino & $13(28.3 \%)$ & $33(71.7 \%)$ & 0.154 \\
\hline Sexco masculino & $21(28.4 \%)$ & $53(71.6 \%)$ & 1 \\
\hline Ventilado & $5(8.3 \%)$ & $55(91.7 \%)$ & 0.0001 \\
\hline Diabetes mellitus 2 & $20(40.8 \%)$ & $29(59.2 \%)$ & 0.012 \\
\hline Hipertensión arterial sistémica & $11(25 \%)$ & $33(75 \%)$ & 0.538 \\
\hline IRC & $5(31.3 \%)$ & $11(68.8 \%)$ & 0.781 \\
\hline Candida Score & 0.29 & 0.56 & 0.035 \\
\hline SIRS & $6(25 \%)$ & $18(75 \%)$ & 0.685 \\
\hline Fiebre & $13(28.3 \%)$ & $33(71.7 \%)$ & 0.989 \\
\hline IRA & $3(8.8 \%)$ & $24(27.9 \%)$ & 0.024 \\
\hline Saturación & 93.21 & 93.44 & 0.766 \\
\hline $\mathrm{FiO}_{2}$ & 29.32 & 48.66 & 0.0001 \\
\hline Edad & 52.56 & 57.79 & 0.158 \\
\hline IMC & 28.7 & 26.8 & 0.066 \\
\hline Creatinina & 0.96 & 1.91 & 0.539 \\
\hline BUN & 24.21 & 30.82 & 0.679 \\
\hline Depuración de creatinina en 24 h & 81.12 & 89.31 & 0.562 \\
\hline Superficie corporal & 1.88 & 1.79 & 0.023 \\
\hline APACHE II & 7.76 & 13.46 & 0.0001 \\
\hline Mortalidad predicha & 7.76 & 13.46 & 0.0001 \\
\hline CPIS & 2.23 & 6.3 & 0.0001 \\
\hline Días de VM & 3.11 & 7.15 & 0.014 \\
\hline
\end{tabular}

Los resultados se expresan en porcentajes o media de los valores.

IMC: índice de masa corporal; IRA: insuficiencia renal aguda; IRC: insuficiencia respiratoria crónica; BUN: nitrógeno ureico en sangre; APACHE II: Acute Physiology And Chronic Health Evaluation II; CPIS: escala de valoración clínica de la infección pulmonar; $\mathrm{FiO}_{2}$ : fracción inspirada de oxigeno; SIRS: respuesta inflamatoria sistémica; VM: ventilación mecánica.

requerimientos de $\mathrm{FiO}_{2}$ (Tabla 3). Por regresión logística se encontró que los niveles bajos de albúmina fueron un factor independiente de mortalidad para este grupo de estudio, con una significancia estadística de $p=0.0001$.

\section{DISCUSIÓN}

La prevalencia de DM2 fue del $40.8 \%$, lo que muestra diferencias con el estudio de referencia ${ }^{10}$, en el que fue de $\sim 18.14 \%$; esto puede deberse a diferencias poblacionales, además de que nuestro hospital es de referencia. El 59.2\% de los pacientes con DM2 presentaba hipoalbuminemia. Se encontró que la supervivencia en los pacientes con niveles normales de albúmina fue del $91.2 \%$; en contraste, en los pacientes con hipoalbuminemia se presentó el mismo número de muertes que de sobrevivientes $(p=0.0001)$. Tanto en el análisis comparativo con $\chi^{2}$ como en el de regresión logística se encontró que los niveles bajos de albúmina fueron un factor independiente de mortalidad. Esto es apoyado por el estudio de Akirov, et al. ${ }^{11}$, en donde encontraron que niveles bajos de albúmina al ingreso al hospital se asociaron con una mayor mortalidad a corto y largo plazos, y la normalización de los niveles de albúmina antes del alta se asoció con un menor riesgo de mortalidad, en comparación con hipoalbuminemia antes del alta.

Respecto a otros factores con asociación significativa con hipoalbuminemia, se encontró que el $91.7 \%$ de los pacientes con hipoalbuminemia se encontraba con ventilación mecánica, lo que concuerda con el estudio de Feng, et al., en donde el seguimiento de una cohorte demostró que la tasa de mortalidad aumentó al 72.1\% para los pacientes > 85 años de edad que habían 
recibido ventilación mecánica durante $>7$ días, relacionando además la edad como factor asociado a mortalidad $^{12}$.

Los pacientes con hipoalbuminemia también presentaron mayor incidencia de IRA. Previamente se ha estudiado la IRA como factor de riesgo para mortalidad en pacientes hospitalizados. Wang, et al. encontraron, en el seguimiento de una cohorte, relación entre la IRA y la mortalidad en pacientes hospitalizados, con una OR de 4.435 (IC 95\%: 3.68-5.35) $)^{13}$.

Como sucedió en nuestro estudio, principalmente respecto a IRA y ventilación mecánica, otras cohortes también han asociado las comorbilidades y severidad de la enfermedad con mayor mortalidad. En el estudio de Mamary, et al. ${ }^{14}$ se asoció la muerte intrahospitalaria con IRA que requiere hemodiálisis, hemodiálisis, insuficiencia cardiaca congestiva, diabetes mellitus, mayor índice de comorbilidad de Charlson, mayor puntuación APACHE II, albúmina inferior y mayor número de transfusiones.

Otros factores independientes que afectaron la mortalidad en nuestro estudio fue la puntuación APACHE II y mayor puntuación CPIS, con promedio de CPIS de 6.6 vs. 4.2, con $p=0.0001$ para los pacientes que fallecieron. Esto es concordante con el estudio de Iribarren, et al. ${ }^{15}$, en donde se encontró que el riesgo relativo (RR) se duplica en los pacientes más graves (coma al ingreso, fármacos vasoactivos, enfermedades previas, APACHE $\geq 22$ ).

\section{CONCLUSIONES}

Las condiciones metabólicas se asociaron a mortalidad en los pacientes hospitalizados con neumonía nosocomial; en efecto, la diabetes mellitus tipo 2 se asoció con una mayor proporción de hipoalbuminemia, lo que predispone a mayor morbimortalidad. La hipoalbuminemia fue un factor independiente de mortalidad, con significancia estadística, y se asoció a variables como días de ventilación mecánica, mayores puntuaciones de APACHE II e IRA.

\section{BIBLIOGRAFÍA}

1. Gatta A, Verardo A, Bolognesi M. Hypoalbuminemia. Int Emerg Med. 2012;7(S3):193-9.

2. Bae JC, Seo SH, Hur KY, Kim JH, Lee MS, Lee MK, et al. Association between serum albumin, insulin resistance, and incident diabetes in nondiabetic subjects. Endocr Metab. 2013;28(1):26-32.

3. Nicholson JP, Wolmarans MR, Park GR. The role of albumin in critical illness. Br J Anaesth. 2000;85(4):599-610.

4. Moctezuma MY, Rodríguez LL, Parra RJA. Asociación de albúmina sérica con severidad de retinopatía diabética. Med Int Mex. 2012;28(3):213-9.

5. Franch-Arcas $\mathrm{G}$. The meaning of hypoalbuminaemia in clinical practice. Clin Nutr. 2001;20:265-9

6. Brock F, Bettinelli LA, Dobner T, Stobbe JC, Pomatti G, Telles CT. Prevalencia de hipoalbuminemia y aspectos nutricionales en ancianos hospitalizados. Rev Latinoamer Enfermagen. 2016;24:e2736.

7. Bonilla-Palomas JL, Gámez-López AL, Moreno-Conde M, López-Ibáñez MC, Anguita-Sánchez M, Gallego de la Sacristana A, et al. Hypoalbuminemia in acute heart failure patients: Causes and its impact on hospital and long-term mortality. J Card Fail. 2014;20(5):350-8

8. Shlipak MG, Fried LF, Crump C, Bleyer AJ, Manolio TA, Tracy RP, et al. Elevations of inflammatory and procoagulant biomarkers in elderly persons with renal insufficiency. Circulation. 2003,107:87-92.

9. Papagianni A, Kalovoulos M, Kirmizis D, Vainas A, Belechri AM, AlexopouIos $\mathrm{E}$, et al. Carotid atherosclerosis is associated with inflammation and endothelial cell adhesion molecules in chronic hemodialysis patients. Nephrol Dial Transplant. 2003;18:113-9.

10. Gutiérrez Cortizo E, Romero Jiménez M, Melero Ruiz B, Massi Aguirre $P$, Fernández Soto J, Pérez Tristancho M, et al. Prevalencia de diabetes mellitus en pacientes hospitalizados. Comunicación XXXV Congreso Nacional de la Sociedad Española de Medicina Interna (SEMI). Murcia, España, 19-21 noviembre 2004. Disponible en: https://www.revclinesp.es/es-congresosxxxv-congreso-nacional-sociedad-espanola-15-sesion-diabetes-1357-prevalencia-de-diabetes-mellitus-en-14999

11. Akirov A, Masri-Iraqi H, Atamna A, Shimon I. Low albumin levels are associated with mortality risk in hospitalized patients. Am J Med. 2017; 130(12):1465.e11-1465.e19.

12. Feng $Y$, Amoateng-Adjepong $Y$, Kaufman D, Gheorghe C, Manthous CA. Age, duration of mechanical ventilation and outcomes of patients who are critically ill. Chest. 2009;136(3):759-64.

13. Wang HE, Muntner P, Chertow GM, Warnock DG. Acute kidney injury and mortality in hospitalized patients. Am J Nephrol. 2012;35:349-55.

14. Mamary AJ, Kondapaneni S, Vance GB, Gaughan JP, Martin UJ, Criner GJ. Survival in patients receiving prolonged ventilation: Factors that influence outcome. Clin Med Insights Circ Respir Pulm Med. 2011;5:17-26.

15. Iribarren BO, Aranda TJ, Dorn HL, Ferrada MM, Ugarte EHA, Koscina MV, et al. Factores de riesgo para mortalidad en neumonía asociada a ventilación mecánica. Rev Chi Infect. 2009;26(3);227-32. 